\title{
Asymptotic Neutrality of Large Ions
}

\author{
Charles L. Fefferman ${ }^{\star}$ and Luis A. Seco $\star$ \\ Department of Mathematics, Princeton University, Princeton, NJ 08544, USA
}

\begin{abstract}
It is proved that a nucleus of charge $Z$ can bind at most $Z+O\left(Z^{a}\right)$ electrons, with $a=47 / 56$.
\end{abstract}

Consider the Hamiltonian for a nucleus of charge $Z$ and $N$ quantized electrons,

$$
H_{Z, N}=\sum_{i=1}^{N}\left[\left(-\Delta_{x_{i}}\right)-\frac{Z}{\left|x_{i}\right|}\right]+\frac{1}{2} \sum_{i \neq j} \frac{1}{\left|x_{i}-x_{j}\right|}=-\Delta+V_{\text {Coulomb }} .
$$

The ground state energy is then

$$
E(Z)=\inf _{N} E(Z, N)=\inf _{N} \inf _{\substack{\psi \in \mathscr{*} \\\|\|_{2}=1}}\left\langle H_{Z, N} \psi, \psi\right\rangle,
$$

where $\mathscr{H}=\bigwedge_{i=1}^{N}\left(L^{2}\left(\mathbf{R}^{3}\right) \otimes \mathbf{C}^{q}\right)$ is the space of antisymmetric wave functions with $q$ spins. Throughout this paper we will simply refer to them as "antisymmetric" wave functions.

For each $Z$, call $N(Z)$ the smallest number for which $E(Z)=E(Z, N)$. It is an interesting problem to obtain sharp estimates for $N(Z)$. The sharpest known result appears in [8], where the reader will find a discussion of the history of the problem. In particular, $N(Z) / Z \rightarrow 1$ as $Z \rightarrow \infty$, although there were no estimates for the rate of convergence. Our main result is the following:

\section{Theorem.}

$$
N(Z)=Z+O\left(Z^{\alpha}\right) \text { for } \quad \alpha=\frac{47}{56} .
$$

We announced this result in [1]. We are grateful to V. Bach for pointing out a

* Partially supported by a NSF grant at Princeton University

$\star \star$ Supported by a Sloan Foundation Dissertation Fellowship at Princeton University 
minor error in our announcement. The rest of the paper is devoted to the proof of this theorem.

For the proof we will be interested only in the case $Z \leqq N \leqq 2 Z$. Lieb ([6] and [7]) has given a simple argument to handle the case $N>2 Z$. Thomas-Fermi theory will play a central role in the proof. We recall a few fundamental facts. For a nice detailed discussion see [5].

1. $E(Z) \sim C_{\mathrm{TF}} Z^{7 / 3}$ as $Z \rightarrow \infty$.

2. Let $\rho_{\mathrm{TF}}$ be Thomas-Fermi density. Then:

a. $\int_{\mathbf{R}^{3}} \rho_{\mathrm{TF}}(x) d x=Z$.

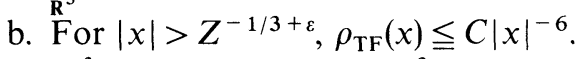

c. $\int_{|x|>R} \rho_{\mathrm{TF}}(x) d x \leqq C_{\rho \mathrm{TF}} R^{-3}$ for $R>Z^{-1 / 3}$, for some constant $C_{\rho \mathrm{TF}}$.

\section{Key Estimate}

Fix $N$ points in $\mathbf{R}^{3}, x_{1}, \ldots, x_{N}$. Take a radially symmetric function $\phi$, supported in $B\left(0, \frac{1}{100} Z^{-2 / 3}\right), \int \phi=1$, sup $|\phi| \leqq C Z^{2}$, and set $\rho_{x_{1}, \ldots, x_{N}}(x)=\sum_{i} \phi\left(x-x_{i}\right)$. Observe that the subharmonicity and positive-definiteness of the Coulomb potential implies that

$$
\begin{aligned}
\sum_{i<j} \frac{1}{\left|x_{i}-x_{j}\right|} \geqq & \frac{1}{2} \iint \frac{\rho_{x_{1}, \ldots, x_{N}}(x) \rho_{x_{1}, \ldots, x_{N}}(y)}{|x-y|} d x d y-C Z^{2 / 3} \cdot N \\
= & \frac{1}{2} \iint \frac{\left(\rho_{x_{1}, \ldots, x_{N}}-\rho_{\mathrm{TF}}\right)(x)\left(\rho_{x_{1}, \ldots, x_{N}}-\rho_{\mathrm{TF}}\right)(y)}{|x-y|} d x d y \\
& +\iint \frac{\rho_{x_{1}, \ldots, x_{N}}(x) \rho_{\mathrm{TF}}(y)}{|x-y|} d x d y-\frac{1}{2} \iint \frac{\rho_{\mathrm{TF}}(x) \rho_{\mathrm{TF}}(y)}{|x-y|} d x d y-C Z^{2 / 3} \cdot N \\
= & c \int|\xi|^{-2}\left|\hat{\rho}_{x_{1}, \ldots, x_{N}}(\xi)-\hat{\rho}_{\mathrm{TF}}(\xi)\right|^{2} d \xi \\
& +\sum_{j} W\left(x_{j}\right)-\frac{1}{2} \iint \frac{\rho_{\mathrm{TF}}(x) \rho_{\mathrm{TF}}(y)}{|x-y|} d x d y-C / Z^{2 / 3} \cdot N
\end{aligned}
$$

with

$$
W(x)=\iint \frac{\phi(x-z) \rho_{\mathrm{TF}}(y)}{|z-y|} d z d y \text {. }
$$

Let's set

$$
K\left(x_{1}, \ldots, x_{N}\right)=\frac{1}{2} \iint \frac{\left(\rho_{x_{1}, \ldots, x_{N}}-\rho_{\mathrm{TF}}\right)(x)\left(\rho_{x_{1}, \ldots, x_{N}}-\rho_{\mathrm{TF}}\right)(y)}{|x-y|} d x d y
$$

and note that $K \geqq 0$ pointwise. This provides the operator inequality

$$
H_{Z, N} \geqq K+\sum_{i=1}^{N}\left[\left(-\Delta_{x_{i}}\right)-\frac{Z}{\left|x_{i}\right|}+W\left(x_{i}\right)\right]-\frac{1}{2} \iint \frac{\rho_{\mathrm{TF}}(x) \rho_{\mathrm{TF}}(y)}{|x-y|} d x d y-C Z^{5 / 3}
$$


for $N \leqq 2 Z$. We point out that similar inequalities were used in [2] and [4].

Let $\lambda_{1}, \lambda_{2}, \ldots$ be the negative eigenvalues of

$$
\left(-\Delta_{x}\right)-\frac{Z}{|x|}+W(x)
$$

and let

$$
\tilde{E}(Z)=q \sum_{i=1}^{\infty} \lambda_{i}-\frac{1}{2} \iint \frac{\rho_{\mathrm{TF}}(x) \rho_{\mathrm{TF}}(y)}{|x-y|} d x d y .
$$

It follows then that

$$
H_{Z, N} \geqq \tilde{E}(Z)+O\left(Z^{5 / 3}\right)
$$

for all $N$ between $Z$ and $2 Z$. From [3] we know that for some $b$ between $1 / 3$ and $2 / 3$ we have

$$
\tilde{E}(Z) \geqq C_{\mathrm{TF}} Z^{7 / 3}+\frac{q}{8} Z^{2}+O\left(Z^{7 / 3-b}\right) .
$$

A careful exposition of Hughes' proof appears in [10]. Similarly, it follows from [7] that

$$
E(Z, N) \leqq C_{\mathrm{TF}} Z^{7 / 3}+\frac{q}{8} Z^{2}+O\left(Z^{7 / 3-b}\right)
$$

for all $N \geqq Z$.

Putting all this together we see that

$$
\tilde{E}(Z) \geqq E(Z, N)+O\left(Z^{7 / 3-b}\right)
$$

for any $N$ between $Z$ and $2 Z$. In particular, we have

$$
H_{Z, N} \geqq E_{0}(Z)+K\left(x_{1}, \ldots, x_{N}\right)-C_{\mathrm{HSW}}\left(Z^{7 / 3-b}\right)
$$

for

$$
E_{0}(Z)=\inf _{N \leqq\left(1+\varepsilon^{\#}\right) Z} E(Z, N),
$$

where $\varepsilon^{\#}$ will be picked later, and some constant $C_{\mathrm{HSw}}$. The constant $b$ plays a crucial role in the analysis of the best possible power of $Z$ for the excess charge. From [3] and [7] it follows that we can take $b=\frac{3}{8}$ : this implies that we can take $\alpha=\frac{47}{56}$ in the statement of the theorem. Notice however that this value of $b$ has been obtained using a much stronger result, namely the correct asymptotics for the energy. It is clear that one can do better and may be one can take $b=\frac{2}{3}$, which would allow us to take $\alpha=\frac{5}{7}$.

\section{Estimates for a Ball}

What we are planning to do now is conclude that if the number of electrons a particular state puts inside a ball is too different from what Thomas-Fermi theory predicts, then this state will have too much energy. This will be then generalized 
to random variables other than the number of electrons inside a ball. We need a few definitions.

Consider a ball $B(0, R)$. Define

$$
N_{R}\left(x_{1}, \ldots, x_{N}\right)=\text { number of } x_{i} \in B(0, R) \text {. }
$$

Also, take a smooth function

$$
\chi=\left\{\begin{array}{lll}
1 & \text { for } & |x|<\frac{4}{3} R \\
0 & \text { for } & |x|>\frac{5}{3} R
\end{array}\right.
$$

that we will call $\chi_{R}$ whenever we want to make it explicit which $R$ is being used, and let

$$
N_{\chi}\left(x_{1}, \ldots, x_{N}\right)=\sum_{i} \phi * \chi\left(x_{i}\right)=\int \rho_{x_{1}, \ldots, x_{N}}(x) \cdot \chi(x) d x .
$$

Observe that $N_{R / 2}\left(x_{1}, \ldots, x_{N}\right) \leqq N_{\chi}\left(x_{1}, \ldots, x_{N}\right) \leqq N_{2 R}\left(x_{1}, \ldots, x_{N}\right)$, for all $x_{1}, \ldots, x_{N}$, provided $R>2 Z^{-2 / 3}$, which will certainly hold in our case, since we will be working with $R \geqq Z^{-1 / 3}$.

Define

$$
N_{\chi}^{\mathrm{TF}}=\int \rho_{\mathrm{TF}}(x) \chi(x) d x
$$

Now, note that

$$
N_{\chi}\left(x_{1}, \ldots, x_{N}\right)-N_{\chi}^{\mathrm{TF}}=\int\left(\rho_{x_{1}, \ldots, x_{N}}-\rho_{\mathrm{TF}}\right)(x) \chi(x) d x=\int\left(\hat{\rho}_{x_{1}, \ldots, x_{N}}-\hat{\rho}_{\mathrm{TF}}\right)(\xi) \hat{\chi}(\xi) d \xi .
$$

Hence

$$
\left|N_{\chi}-N_{\chi}^{\mathrm{TF}}\right|^{2} \leqq \int|\hat{\chi}(\xi)|^{2} \cdot|\xi|^{2} d \xi \int\left|\left(\hat{\rho}_{x_{1}, \ldots, x_{N}}-\hat{\rho}_{\mathrm{TF}}\right)\right|^{2} \cdot|\xi|^{-2} d \xi \leqq C R \cdot K\left(x_{1}, \ldots, x_{N}\right) .
$$

Therefore, (1) implies

$$
\left\langle H_{Z, N} \psi, \psi\right\rangle \geqq E_{0}(Z)+C_{K} \frac{\left\langle\left|N_{\chi}-N_{\chi}^{\mathrm{TF}}\right|^{2} \psi, \psi\right\rangle}{R}-C_{\mathrm{HSW}}\left(Z^{7 / 3-b}\right)
$$

for some constant $C_{K}$. In particular, Cauchy-Schwarz implies

$$
\left\langle H_{Z, N} \psi, \psi\right\rangle \geqq E_{0}(Z)+C_{K} \frac{\left|\left\langle N_{\chi} \psi, \psi\right\rangle-N_{\chi}^{\mathrm{TF}}\right|^{2}}{R}-C_{\mathrm{HSW}}\left(Z^{7 / 3-b}\right)
$$

for any antisymmetric $\psi,\|\psi\|_{2}=1$.

The previous argument can be generalized to yield the following result:

Lemma 2.1. Given any function $\varphi(x) \in L^{2}\left(\mathbf{R}^{3}\right)$, we have

$$
\left\langle H_{Z, N} \psi, \psi\right\rangle \geqq E_{0}(Z)+C_{K} \frac{\left|\left\langle\left(\rho_{\psi} * \phi-\rho_{\mathrm{TF}}\right), \varphi\right\rangle\right|^{2}}{\|\nabla \varphi\|_{2}^{2}}-C_{\mathrm{HSW}}\left(Z^{7 / 3-b}\right)
$$

for $\psi \in \mathscr{H},\|\psi\|_{2}=1$. Here,

$$
\rho_{\psi}=N \int\left|\psi\left(x, x_{2}, \ldots, x_{N}\right)\right|^{2} d x_{2} \cdots d x_{N}
$$


in the case of a fully antisymmetric $\psi($ i.e. $q=1)$ and in general

$$
\rho_{\psi}(x)=\sum_{i=1}^{N} \int\left|\psi\left(x_{1}, \ldots, x_{i-1}, x, x_{i+1}, \ldots, x_{N}\right)\right|^{2} \prod_{i \neq j} d x_{j} .
$$

Now, we define a working variant of estimate (A).

Definition. We say that Estimate $(\bar{\varepsilon}, \varepsilon, R)$ holds if for a nucleus of charge $Z$ at the origin and $N$ quantized electrons confirmed to the ball $B(0, R)$ we have

$$
\left\langle H_{Z, N} \psi, \psi\right\rangle \geqq E_{0}(Z)+\frac{\bar{\varepsilon} Z}{R}(N-(1+\varepsilon) Z)
$$

for normalized $\psi$, where

$$
E_{0}(Z)=\inf _{0 \leqq N \leqq\left(1+\varepsilon^{\#}\right) Z} E(Z, N)
$$

for $\varepsilon^{\#}$ to be picked later. By $N$ quantized electrons confined to the ball $B(0, R)$ we mean that $\psi=\psi\left(x_{1}, \ldots, x_{N}\right)$ is supported in the set $x_{i} \in B(0, R)$ for all $i=1, \ldots, N$.

Now it will be necessary to introduce two parameters $\gamma_{1}$ and $\gamma_{2}$, in the proof. They are related to $b$ by the relation

1. $\gamma_{1}=\frac{3}{7} b$.

2. $\gamma_{2}=b / 7$.

The significance of $\gamma_{1}$ is that it represents the excess charge. Precisely

$$
N(Z)=Z+O\left(Z^{1-\gamma_{1}}\right)
$$

On the other hand, $\gamma_{2}$ is related to the radius of the largest ball for which we can obtain favourable estimates for its excess charge. This is clearly seen in the following lemma.

Lemma 2.2. There exist constants $C_{0}$ and $c_{0}$ independent of $Z$, such that Estimate $(\bar{\varepsilon}, \varepsilon, R)$ holds for

1. $\varepsilon^{\#} \geqq \varepsilon \geqq C_{0} Z^{-\gamma_{1}}$,

2. $\bar{\varepsilon} \leqq c_{0} \bar{Z}^{-\gamma_{1}}$

3. $R \leqq C_{0} Z^{-1 / 3+\gamma_{2}}$.

Proof. Pick $R, \varepsilon$ and $\bar{\varepsilon}$ within this range. Say $\psi$ confines $N$ electrons to $B(0, R)$. If $N \leqq(1+\varepsilon) Z$, then

$$
\begin{aligned}
\left\langle H_{Z, N} \psi, \psi\right\rangle \geqq E(Z, N) & \geqq E_{0}(Z) \\
& \geqq E_{0}(Z)+\frac{\bar{\varepsilon} Z}{R}(N-(1+\varepsilon) Z) .
\end{aligned}
$$

If $N>(1+\varepsilon) Z$, then

$$
\left\langle N_{\chi} \psi, \psi\right\rangle>(1+\varepsilon) Z
$$


for $\chi=\chi_{2 R}$. Since $N_{\chi}^{\mathrm{TF}}<Z$,

$$
\begin{aligned}
C_{K} \frac{\left|\left\langle N_{\chi} \psi, \psi\right\rangle-N_{\chi}^{\mathrm{TF}}\right|^{2}}{2 R} & >C_{K} \frac{|N-Z|^{2}}{2 R}>C_{K} \frac{\varepsilon^{2} Z^{2}}{2 R} \\
& >\frac{C_{K} C_{0}^{2}}{2 C_{0}} Z^{7 / 3-\left(2 \gamma_{1}+\gamma_{2}\right)} \geqq 2 C_{\mathrm{HSW}} Z^{7 / 3-b}
\end{aligned}
$$

for $C_{0}$ large enough; so,

$$
\left\langle H_{Z, N} \psi, \psi\right\rangle \geqq E_{0}(Z)+C_{K} \frac{|N-Z|^{2}}{4 R} .
$$

On the other hand,

$$
C_{K} \frac{|N-Z|^{2}}{4 R}>\frac{\bar{\varepsilon} Z}{R}(N-(1-\varepsilon) Z) \text { for } \quad N \geqq(1+\varepsilon) Z .
$$

To see this, observe that it holds trivially at $N=(1+\varepsilon) Z$, because the right-hand side is zero; if now we differentiate both sides with respect to $N$, we obtain for the left-hand side

$$
\frac{C_{K}(N-Z)}{2 R}>\frac{C_{K}}{2} \cdot \frac{\varepsilon Z}{R}
$$

and $\bar{\varepsilon} Z / R$ for the right-hand side. So Estimate $(\bar{\varepsilon}, \varepsilon, R)$ holds taking $c_{0}$ small enough.

Throughout the proof we will need a couple more conditions on $C_{0}$ and $c_{0}$ that will force us to take them to be larger and smaller respectively than what we needed for this lemma.

Lemma 2.3. Let $R=Z^{-1 / 3+\gamma_{2}}$, and $\chi=\chi_{R}$. Say that for a number $Y$,

$$
\left\langle N_{\chi} \psi, \psi\right\rangle=Z+Y Z^{1-\gamma_{1}} \text {. }
$$

Then, for some universal constant $c_{1}$,

$$
\left\langle H_{Z, N} \psi, \psi\right\rangle \geqq E_{0}(Z)+C_{K} \frac{\left(|Y|-c_{1}\right)_{+}^{2} Z^{2-2 \gamma_{1}}}{4 R}-C_{\mathrm{HSW}} Z^{7 / 3-b} .
$$

Proof. Observe that we have

$$
N_{\chi}^{\mathrm{TF}} \geqq Z-\int_{|x| \geqq R / 2} \rho_{\mathrm{TF}}(x) d x \geqq Z-8 C_{\rho \mathrm{TF}} Z^{1-3 \gamma_{2}}=Z-8 C_{\rho \mathrm{TF}} Z^{1-\gamma_{1}}
$$

and $N_{\chi}^{\mathrm{TF}} \leqq Z$. Thus,

$$
\frac{\left|\left\langle N_{\chi} \psi, \psi\right\rangle-N_{\chi}^{\mathrm{TF}}\right|^{2}}{R} \geqq \frac{\left(\left\langle N_{\chi} \psi, \psi\right\rangle-N_{\chi}^{\mathrm{TF}}\right)_{+}^{2}}{R} \geqq \frac{(Y)_{+}^{2} Z^{2-2 \gamma_{1}}}{R}
$$

and

$$
\frac{\left|\left\langle N_{\chi} \psi, \chi\right\rangle-N_{\chi}^{\mathrm{TF}}\right|^{2}}{R} \geqq \frac{\left(N_{\chi}^{\mathrm{TF}}-\left\langle N_{\chi} \psi, \psi\right\rangle\right)_{+}^{2}}{R} \geqq \frac{\left(-Y-8 C_{\rho \mathrm{TF}}\right)_{+}^{2} Z^{2-2 \gamma_{1}}}{R} .
$$

Applying (A) to both cases and averaging the resulting inequalities, we get

$$
\left\langle H_{Z, N} \psi, \psi\right\rangle \geqq E_{0}(Z)+C_{K} Z^{2-2 \gamma_{1}} \frac{\left(-Y-8 C_{\rho \mathrm{TF}}\right)_{+}^{2}+(Y)_{+}^{2}}{2 R}-C_{\mathrm{HSW}} Z^{7 / 3-b} .
$$


Elementary calculus says that

$$
(-x-c)_{+}^{2}+(x)_{+}^{2} \geqq \frac{\left(|x|-c^{\prime}\right)_{+}^{2}}{2},
$$

and this implies the conclusion of the lemma.

The previous estimates could have been done for $R$ of the form $Z^{-1 / 3+\gamma}$ for $\gamma \leqq \gamma_{2}$, with the only effect of decreasing $\gamma_{1}$ and thus worsening a little the estimates for the excess charge. However, they cannot be carried out with these techniques for $\gamma>\gamma_{2}$, since whatever term we want to estimate will give a contribution so small to the energy that it will simply be lost in the $O\left(Z^{7 / 3-b}\right)$. For radii this big we need a different approach in which we use in a more direct way the properties of the Coulomb potential. The goal of the next section is to analyze how estimates for a given ball imply corresponding estimates for its double.

\section{Estimates for Spherical Shells}

In this section we consider a system of $N$ quantized electrons confined to $B(0, R)$, and $N^{\prime}$ electrons confined to $B(0,2 R)-B(0, R / 2)$. That is, we will be considering wave functions $\psi\left(x_{1}, \ldots, x_{N}, x_{1}^{\prime}, \ldots, x_{N^{\prime}}^{\prime}\right)$ supported on the set $x_{1}, \ldots, x_{N} \in B(0, R)$, $x_{1}^{\prime}, \ldots, x_{N^{\prime}}^{\prime} \in B(0,2 R)-B(0, R / 2)$. We have to impose the extra condition that for fixed $x_{1}^{\prime}, \ldots, x_{N^{\prime}}^{\prime}, \psi$ is antisymmetric in the $x_{1}, \ldots, x_{N}$ and viceversa: that is, we will be considering vector-valued $\psi$, with domain $x_{1}^{\prime}, \ldots, x_{N^{\prime}}^{\prime} \in B(0,2 R)-B(0, R / 2)$, and values in $\mathscr{H}$ and vector-valued $\psi$, with domain $x_{1}, \ldots, x_{N} \in B(0, R)$, and values in $\mathscr{H}$.

To stress the different role of the two sets of electrons, we rewrite the hamiltonian as

$$
H_{Z, N+N^{\prime}}=H_{Z, N}+H_{\text {extra }}=\left(-\Delta_{x_{1}, \ldots, x_{N}}+V\right)+\left(-\Delta_{\text {extra }}+V_{\text {extra }}\right)
$$

with

$$
\begin{aligned}
\Delta_{\text {extra }} & =\Delta_{x_{1}^{\prime}, \ldots, x_{N^{\prime}}^{\prime}}, \\
V & =-\sum_{i=1, \ldots, N} \frac{Z}{\left|x_{i}\right|}+\frac{1}{2} \sum_{i \neq j} \frac{1}{\left|x_{i}-x_{j}\right|}, \\
V_{\text {extra }} & =-\sum_{i=1, \ldots, N^{\prime}} \frac{N}{\left|x_{i}^{\prime}\right|}+\sum_{\substack{i=1, \ldots, N^{\prime} \\
j=1, \ldots, N}} \frac{1}{\left|x_{i}^{\prime}-x_{j}\right|}+\frac{1}{2} \sum_{\substack{i=1, \ldots, N^{\prime} \\
j=1, \ldots, N^{\prime} \\
i \neq j}} \frac{1}{\left|x_{i}^{\prime}-x_{j}^{\prime}\right|} .
\end{aligned}
$$

Also, we restrict our attention to the case $R>C_{0} R_{*}=C_{0} Z^{-1 / 3+\gamma_{2}}$.

The content of the following lemma is as follows: We know from previous estimates that approximately $Z$ of the electrons will organize themselves to be close to the nucleus; this will have the important effect of "screening" the nucleus. That is, all the other electrons will hardly feel any negative electrostatic potential; this has as a consequence that a lot more than $Z$ electrons will only make the energy of the system grow above the ground state energy.

Lemma 3.1. Assume that $N+N^{\prime} \geqq(1+\delta) Z$ for

$$
C_{0} Z^{-\gamma_{1}} \leqq \delta<\left(1+10^{-6}\right) C_{0} Z^{-\gamma_{1}} \text {. }
$$


Then, for some constant $c$,

$$
\left\langle V_{\text {extra }} \psi, \psi\right\rangle \geqq \frac{c \delta Z}{R} N^{\prime}
$$

or else

$$
\left\langle H_{Z, N+N^{\prime}} \psi, \psi\right\rangle \geqq E_{0}(Z)+c Z^{7 / 3-7 \gamma_{2}}
$$

Proof. We will assume that $N^{\prime} \neq 0$ or else there is nothing to prove. Recall that $R_{*}=Z^{-1 / 3+\gamma_{2}}$. Let $\chi=\chi_{R_{*}}$. Note that

$$
N_{\chi}=\sum_{i=1, \ldots, N} \chi * \phi\left(\chi_{i}\right)+\sum_{j=1, \ldots, N^{\prime}} \chi * \phi\left(x_{j}^{\prime}\right)=\sum_{i=1, \ldots, N} \chi * \phi\left(x_{i}\right)
$$

as operators on our space of functions, since $\chi * \phi$ and $\psi$ have disjoint support in the $x_{j}^{\prime}$ variables.

Let $V_{\text {extra }}=\sum_{j} V_{j}\left(x_{1}, \ldots, x_{N}, x_{1}^{\prime}, \ldots, x_{N^{\prime}}^{\prime}\right)$ for

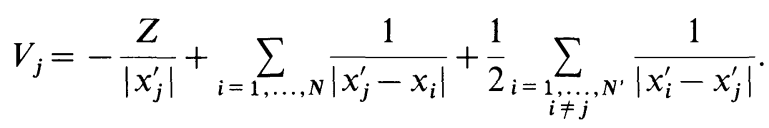

Note that

$$
\begin{aligned}
\sum_{i=1}^{N} \frac{1}{\left|x_{j}^{\prime}-x_{i}\right|} & =\sum_{i=1}^{N} \frac{\chi * \phi\left(x_{i}\right)}{\left|x_{j}^{\prime}-x_{i}\right|}+\sum_{i=1}^{N} \frac{1-\chi * \phi\left(x_{i}\right)}{\left|x_{j}^{\prime}-x_{i}\right|} \\
-\frac{Z}{\left|x_{j}^{\prime}\right|} & =-\frac{Z-N_{\chi}}{\left|x_{j}^{\prime}\right|}-\sum_{i=1}^{N} \frac{\chi * \phi\left(x_{i}\right)}{\left|x_{j}^{\prime}\right|}
\end{aligned}
$$

and write

$$
\begin{aligned}
V_{j}\left(x_{1}, \ldots, x_{N}, x_{1}^{\prime}, \ldots, x_{N^{\prime}}^{\prime}\right)= & -\frac{Z-N_{\chi}}{\left|x_{j}^{\prime}\right|}+\sum_{i=1}^{N} \frac{1-\chi * \phi\left(x_{i}\right)}{\left|x_{j}^{\prime}-x_{i}\right|} \\
& +\frac{1}{2} \sum_{i=1, \ldots, N^{\prime}} \frac{1}{\left|x_{j}^{\prime}-x_{i}^{\prime}\right|}+\sum_{i=1}^{N}\left(\frac{1}{\left|x_{j}^{\prime}-x_{i}\right|}-\frac{1}{\left|x_{j}^{\prime}\right|}\right) \cdot(\chi * \phi)\left(x_{i}\right) \\
= & T_{1}+T_{2}+T_{3}+T_{4} .
\end{aligned}
$$

Let's analyze this term by term:

$\cdot)\left\langle T_{1} \psi, \psi\right\rangle \geqq-\frac{\left|Z-\left\langle N_{\chi} \psi, \psi\right\rangle\right|}{R / 2}$.

.) $T_{2} \geqq \frac{N-N_{\chi}}{8 R}$ pointwise.

$\cdot)\left\langle T_{3} \psi, \psi\right\rangle \geqq \frac{N^{\prime}-1}{8 R}$. 
Therefore

$$
\begin{aligned}
\left\langle\left(T_{1}+T_{2}+T_{3}\right) \psi, \psi\right\rangle & \geqq \frac{N+N^{\prime}-\left\langle N_{\chi} \psi, \psi\right\rangle-16\left|Z-\left\langle N_{\chi} \psi, \psi\right\rangle\right|-1}{8 R} \\
& \geqq \frac{\delta Z-20\left|Z-\left\langle N_{\chi} \psi, \psi\right\rangle\right|+\Omega}{9 R}
\end{aligned}
$$

for $\Omega=N+N^{\prime}-\delta Z-Z$. Note that by hypothesis, $\Omega \geqq 0$. Summing over all $j$ we obtain

$$
\sum_{j=1}^{N^{\prime}}\left\langle\left(T_{1}+T_{2}+T_{3}\right) \psi, \psi\right\rangle \geqq \frac{\delta Z-20\left|Z-\left\langle N_{\chi} \chi, \psi\right\rangle\right|+\Omega}{9 R} N^{\prime} .
$$

If we could prove that $\left|Z-\left\langle N_{\chi} \psi, \psi\right\rangle\right|\left\langle c Z^{1-\gamma_{1}}\right.$ for some constant independent of $C_{0}$, then we would have

$$
\left\langle\left(T_{1}+T_{2}+T_{3}\right) \psi, \psi\right\rangle \geqq \frac{\delta Z-20\left|Z-\left\langle N_{\chi} \psi, \psi\right\rangle\right|+\Omega}{9 R} \geqq \frac{\delta Z}{10 R}
$$

by simply taking $C_{0}$ large enough. So, the result will follow if we can prove that either

$$
\left\langle H_{Z, N+N^{\prime}} \psi, \psi\right\rangle \geqq E_{0}(Z)+c Z^{7 / 3-7 \gamma_{2}}
$$

or else:

$$
\left|\sum_{j}\left\langle T_{4} \cdot \psi, \psi\right\rangle\right|<\frac{\delta Z}{16 R} N^{\prime}
$$

and

$$
\left|Z-\left\langle N_{\chi} \psi, \psi\right\rangle\right| \leqq c Z^{1-\gamma_{1}}
$$

with $c$ independent of $C_{0}$.

In order to analyze this, let's define

$$
\psi_{x_{1}^{\prime}, \ldots, x_{N^{\prime}}^{\prime}}\left(x_{1}, \ldots, x_{N}\right)=\psi\left(x_{1}, \ldots, x_{N}, x_{1}^{\prime}, \ldots, x_{N^{\prime}}^{\prime}\right)
$$

together with its normalized version

$$
\bar{\psi}_{x_{1}^{\prime}, \ldots, x_{N}^{\prime}}\left(x_{1}, \ldots, x_{N}\right)=\left\|\psi_{x_{1}^{\prime}, \ldots, x_{N}^{\prime}}\right\|_{L^{2}\left(d x_{1} \cdots d x_{N}\right)}^{-1} \psi_{x_{1}^{\prime}, \ldots, x_{N^{\prime}}^{\prime}}\left(x_{1}, \ldots, x_{N}\right),
$$

defined on the set

$$
E=\left\{\left(x_{1}^{\prime}, \ldots, x_{N^{\prime}}^{\prime}\right) \mid\left\|\psi_{x_{1}^{\prime}, \ldots, x_{N}^{\prime}}\right\|_{2} \neq 0\right\} .
$$

Also, as in Lemma 2.1, define

$$
\begin{aligned}
& \rho_{\psi_{x_{1}^{\prime}, \ldots, r_{N}^{\prime}}}(y)=\sum_{i=1}^{N} \int\left|\psi\left(x_{1}, \ldots, x_{i-1}, y, x_{i+1}, \ldots, x_{N}, x_{1}^{\prime}, \ldots, x_{N^{\prime}}^{\prime}\right)\right|^{2} \prod_{i \neq j} d x_{j}, \\
& \rho_{\bar{\psi}_{\mathrm{r}_{1}^{\prime}, \ldots, \mathrm{r}_{N^{\prime}}^{\prime}}}(y)=\sum_{i=1}^{N} \int\left|\bar{\psi}\left(x_{1}, \ldots, x_{i-1}, y, x_{i+1}, \ldots, x_{N}, x_{1}^{\prime}, \ldots, x_{N^{\prime}}^{\prime}\right)\right|^{2} \prod_{i \neq j} d x_{j} .
\end{aligned}
$$


Note also that since $\rho_{\mathrm{TF}}$ and $\chi$ are radially symmetric

$$
\int\left(\frac{1}{\left|x_{j}^{\prime}-y\right|}-\frac{1}{\left|x_{j}^{\prime}\right|}\right) \rho_{\mathrm{TF}}(y) \chi(y) d y=0 .
$$

Then

$$
\begin{aligned}
& \int T_{4}\left|\bar{\psi}\left(x_{1}, \ldots, x_{N}, x_{1}^{\prime}, \ldots, x_{N^{\prime}}^{\prime}\right)\right|^{2} d x_{1} \cdots d x_{N} \\
& =\int\left(\frac{1}{\left|x_{j}^{\prime}-y\right|}-\frac{1}{\left|x_{j}^{\prime}\right|}\right)(\chi * \phi)(y) \rho_{\bar{\psi}_{x_{1}^{\prime}, \ldots, x_{N}^{\prime}}}(y) d y \\
& =\iint\left(\frac{1}{\left|x_{j}^{\prime}-y\right|}-\frac{1}{\left|x_{j}^{\prime}\right|}\right) \chi(z) \phi(y-z) \rho_{\bar{\psi}_{x_{1}^{\prime}, \ldots x_{N^{\prime}}^{\prime}}}(y) d y d z \\
& =\iint\left(\frac{1}{\left|x_{j}^{\prime}-z\right|}-\frac{1}{\left|x_{j}^{\prime}\right|}\right) \chi(z) \phi(y-z) \rho_{\bar{\psi}_{r_{1}^{\prime} \ldots, r_{N^{\prime}}^{\prime}}^{\prime}}(y) d y d z \\
& -\iint\left(\frac{1}{\left|x_{j}^{\prime}-z\right|}-\frac{1}{\left|x_{j}^{\prime}-y\right|}\right) \chi(z) \phi(y-z) \rho_{\bar{\psi}_{x_{1}^{\prime} \ldots, x_{N^{\prime}}^{\prime}}}(y) d y d z \\
& =\int\left(\frac{1}{\left|x_{j}^{\prime}-y\right|}-\frac{1}{\left|x_{j}^{\prime}\right|}\right) \chi(y)\left(\phi * \rho_{\bar{\psi}_{\mathrm{x}_{1}^{\prime}, \ldots, \mathrm{r}_{N^{\prime}}}}\right)(y) d y \\
& -\iint\left(\frac{1}{\left|x_{j}^{\prime}-z\right|}-\frac{1}{\left|x_{j}^{\prime}-y\right|}\right) \chi(x) \phi(y-z) \rho_{\bar{\psi}_{x_{1}^{\prime}, \ldots, r_{N^{\prime}}^{\prime}}}(y) d y d z \text {. }
\end{aligned}
$$

Now observe that

$$
\begin{aligned}
& \left|\iint\left(\frac{1}{\left|x_{j}^{\prime}-z\right|}-\frac{1}{\left|x_{j}^{\prime}-y\right|}\right) \chi(z) \phi(y-z) \rho_{\bar{\psi}_{\mathrm{x}_{1}^{\prime}, \ldots, \mathrm{r}_{N^{\prime}}}}(y) d y d z\right| \\
& \quad=\leqq 10 \iint \frac{|z-y|}{R^{2}} \chi(z) \phi(y-z) \rho_{\bar{\psi}_{\mathrm{x}_{1}^{\prime}, \ldots,,_{N^{\prime}}^{\prime}}(y) d y d z} \\
& \quad \leqq 10 N \|\left.\int \frac{|z-y|}{R^{2}} \chi(z) \phi(y-z) d z\right|_{L^{\infty}(d y)} \\
& \quad=N \frac{Z^{-2 / 3}}{R^{2}} \int \phi(z) d z \leqq 2 \frac{Z^{1 / 3}}{R^{2}} .
\end{aligned}
$$

Summing over all $j$ we obtain that

$$
\left|\sum_{j} \iint\left(\frac{1}{\left|x_{j}^{\prime}-z\right|}-\frac{1}{\left|x_{j}^{\prime}-y\right|}\right) \chi(z) \phi(y-z) \rho_{\bar{\psi}_{x_{1}^{\prime}, \ldots, x_{N^{\prime}}^{\prime}}}(y) d y d z\right| \leqq 2 \frac{Z^{1 / 3}}{R^{2}} N^{\prime} .
$$

Note that

$$
\frac{Z^{1 / 3}}{R^{2}} N^{\prime} \leqq \frac{Z^{2 / 3-\gamma_{2}}}{R} N^{\prime} \ll \frac{\delta Z}{R} N^{\prime}
$$

as long as $\frac{2}{3}-\gamma_{2}<1-3 \gamma_{2}$, that is, $\gamma_{2}<\frac{1}{6}$, or $b<\frac{7}{6}$, which certainly holds in this 
case. So

$$
\begin{aligned}
& \left.\left|\sum_{j} \int T_{4}\right| \bar{\psi}\left(x_{1}, \ldots, x_{N}, x_{1}^{\prime}, \ldots, x_{N^{\prime}}^{\prime}\right)\right|^{2} d x_{1} \cdots d x_{N} \mid \\
& \quad \leqq\left|\sum_{j} \int\left(\frac{1}{\left|x_{j}^{\prime}-y\right|}-\frac{1}{\left|x_{j}^{\prime}\right|}\right) \chi(y)\left(\rho_{\bar{\psi}_{\mathrm{x}_{1}^{\prime}, \ldots, \mathrm{r}_{N^{\prime}}^{\prime}}} * \phi\right)(y) d y\right|+2 \frac{Z^{1 / 3}}{R^{2}} N^{\prime} \\
& \quad=\left|\sum_{j} \int\left(\frac{1}{\left|x_{j}^{\prime}-y\right|}-\frac{1}{\left|x_{j}^{\prime}\right|}\right) \chi(y)\left(\rho_{\bar{\psi}_{\mathrm{x}_{1}^{\prime}, \ldots, \mathrm{r}_{N^{\prime}}}} * \phi(y)-\rho_{\mathrm{TF}}(y)\right) d y\right|+2 \frac{Z^{1 / 3}}{R^{2}} N^{\prime} \\
& \quad=\mid\left\langle\sum_{j}\left(\frac{1}{\left|x_{j}^{\prime}-y\right|}-\frac{1}{\left|x_{j}^{\prime}\right|}\right) \cdot \chi,\left(\rho_{\bar{\psi}_{x_{1}^{\prime}, \ldots, r_{N^{\prime}}}} * \phi-\rho_{\mathrm{TF}}\right\rangle\right|+2 \frac{Z^{1 / 3}}{R^{2}} N^{\prime} .
\end{aligned}
$$

So, if we define

$$
\varphi(y)=\sum_{j}\left(\frac{1}{\left|x_{j}^{\prime}-y\right|}-\frac{1}{\left|x_{j}^{\prime}\right|}\right) \cdot \chi(y) .
$$

we have

$\left.\left|\sum_{j} \int T_{4}\right| \bar{\psi}\left(x_{1}, \ldots, x_{N}, x_{1}^{\prime}, \ldots, x_{N^{\prime}}^{\prime}\right)\right|^{2} d x_{1} \cdots d x_{N}|\leqq|\left\langle\varphi,\left(\rho_{\bar{\psi}_{x_{1}, \ldots, x_{N^{\prime}}}} * \phi-\rho_{\mathrm{TF}}\right)\right\rangle \mid+2 \frac{Z^{1 / 3}}{R^{2}} N^{\prime}$

In particular

$$
\begin{aligned}
& \left(\left.\left|\sum_{j} \int T_{4}\right| \bar{\psi}\left(x_{1}, \ldots, x_{N}, x_{1}^{\prime}, \ldots, x_{N^{\prime}}^{\prime}\right)\right|^{2} d x_{1} \cdots d x_{N} \mid-2 \frac{Z^{1 / 3}}{R^{2}} N^{\prime}\right)_{+}^{2} \\
& \quad \leqq\left|\left\langle\varphi,\left(\rho_{\bar{\psi}_{\mathrm{x}_{1}^{\prime} \ldots, \mathrm{r}_{N^{\prime}}}} * \phi-\rho_{\mathrm{TF}}\right)\right\rangle\right|^{2} .
\end{aligned}
$$

Next, observe that

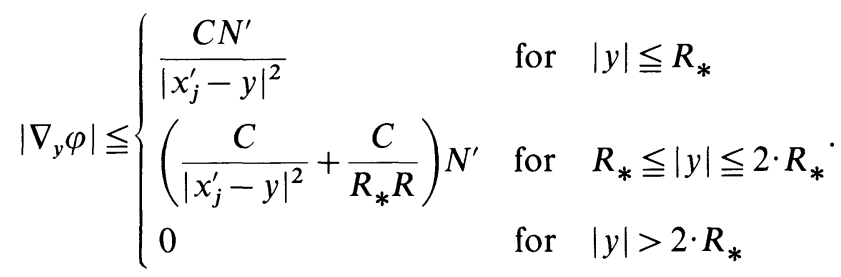

In any case,

$$
\left|\nabla_{y} \varphi\right| \leqq \frac{C N^{\prime}}{R R_{*}}
$$

therefore,

$$
\left\|\nabla_{y} \varphi\right\|_{2}^{2} \leqq C N^{\prime 2} \frac{R_{*}}{R^{2}}
$$

By Lemma 2.1 applied to $\bar{\psi}$, we have for $\left(x_{1}^{\prime}, \ldots, x_{N^{\prime}}^{\prime}\right) \in E$, 


$$
\begin{aligned}
& \left\langle H_{Z, N} \bar{\psi}_{r_{1}^{\prime}, \ldots, x_{N^{\prime}}^{\prime}}, \bar{\psi}_{r_{1}^{\prime}, \ldots, r_{N^{\prime}}^{\prime}}\right\rangle \\
& \geqq E_{0}(Z)+C^{\prime} \frac{R^{2}}{N^{\prime 2} R_{*}}\left|\left\langle\rho_{\bar{\psi}_{r_{1}^{\prime}, \ldots, x_{N^{\prime}}^{\prime}}} * \phi-\rho_{\mathrm{TF}}, \varphi\right\rangle\right|^{2}-C_{\mathrm{HSw}} Z^{7 / 3-b} \\
& \geqq E_{0}(Z)+C^{\prime} \frac{R^{2}}{N^{\prime 2} R_{*}}\left(\left|\left\langle\sum_{j} T_{4} \bar{\psi}_{x_{1}^{\prime}, \ldots, x_{N^{\prime}}^{\prime}}, \bar{\psi}_{\mathrm{r}_{1}^{\prime}, \ldots, x_{N^{\prime}}^{\prime}}\right\rangle\right|-2 \frac{Z^{1 / 3}}{R^{2}} N^{\prime}\right)_{+}^{2}-C_{\mathrm{HSW}} Z^{7 / 3-b} .
\end{aligned}
$$

In other words,

$$
\begin{aligned}
& \left\langle H_{Z, N} \psi_{x_{1}^{\prime}, \ldots, r_{N^{\prime}}^{\prime}}, \psi_{x_{1}^{\prime}, \ldots,,_{N^{\prime}}^{\prime}}\right\rangle \\
& \geqq\left\|\psi_{\mathrm{r}_{1}^{\prime}, \ldots, \mathrm{r}_{N^{\prime}}^{\prime}}\right\|_{2}^{2} \cdot\left(E_{0}(Z)-C_{\mathrm{HSW}} Z^{7 / 3-b}+C^{\prime} \frac{R^{2}}{N^{\prime 2} R_{*}}\right. \\
& \left.\frac{\left.\left(\left|\left\langle\sum_{j} T_{4} \psi_{r_{1}^{\prime}, \ldots, r_{N^{\prime}}^{\prime}}, \psi_{x_{1}^{\prime}, \ldots, x_{N^{\prime}}^{\prime}}\right\rangle\right|-2 \frac{Z^{1 / 3}}{R^{2}} N^{\prime} \cdot\left\|\psi_{x_{1}^{\prime}, \ldots, r_{N^{\prime}}^{\prime}}\right\|_{2}^{2}\right)_{+}^{2}\right)}{\left\|\psi_{r_{1}^{\prime}, \ldots, r_{N^{\prime}}^{\prime}}\right\|_{2}^{4}}\right) \\
& =\left(E_{0}(Z)-C_{\mathrm{HSW}} Z^{7 / 3-b}\right)\left\|\psi_{r_{1}^{\prime}, \ldots, r_{N^{\prime}}^{\prime}}\right\|_{2}^{2} \\
& +C^{\prime} \frac{R^{2}}{N^{\prime 2} R_{*}} \frac{\left(\left|\left\langle\sum_{j} T_{4} \psi_{r_{1}^{\prime}, \ldots, r_{N^{\prime}}^{\prime}}, \psi_{x_{1}^{\prime}, \ldots, r_{N^{\prime}}^{\prime}}\right\rangle\right|-2 \frac{Z^{1 / 3}}{R^{2}} N^{\prime} \cdot\left\|\psi_{r_{1}^{\prime}, \ldots, x_{N^{\prime}}^{\prime}}\right\|_{2}^{2}\right)_{+}^{2}}{\left\|\psi_{r_{1}^{\prime}, \ldots, x_{N^{\prime}}^{\prime}}\right\|_{2}^{2}} .
\end{aligned}
$$

Now, integrate with respect to $\left(x_{1}^{\prime}, \ldots, x_{N^{\prime}}^{\prime}\right) \in E$ to obtain

$$
\begin{aligned}
\left\langle H_{Z, N} \psi, \psi\right\rangle \geqq & E_{0}(Z)-C_{\mathrm{HSW}} Z^{7 / 3-b}+C^{\prime} \frac{R^{2}}{N^{\prime 2} R_{*}} \\
& \cdot \int_{E} \frac{\left(\left|\left\langle\sum_{j} T_{4} \psi_{r_{1}^{\prime}, \ldots, x_{N^{\prime}}^{\prime}}, \psi_{x_{1}^{\prime}, \ldots, x_{N^{\prime}}^{\prime}}\right\rangle\right|-2 \frac{Z^{1 / 3}}{R^{2}} N^{\prime} \cdot\left\|\psi_{x_{1}^{\prime}, \ldots, r_{N^{\prime}}^{\prime}}\right\|_{2}^{2}\right)_{+}^{2}}{\left\|\psi_{r_{1}^{\prime}, \ldots, x_{N^{\prime}}^{\prime}}\right\|_{2}^{2}} d x_{1}^{\prime} \cdots d x_{N^{\prime}}^{\prime} .
\end{aligned}
$$

Now use Cauchy-Schwarz and the fact that

$$
\int(f(x))_{+} d x \geqq\left(\int f(x) d x\right)_{+} \geqq 0
$$

to realize that

$$
\begin{aligned}
& \left(\left|\left\langle\sum_{j} T_{4} \psi, \psi\right\rangle\right|-2 \frac{Z^{1 / 3}}{R^{2}} N^{\prime}\right)_{+}^{2} \\
& \quad \leqq\left(\int_{E}\left(\left|\left\langle\sum_{j} T_{4} \psi_{x_{1}^{\prime}, \ldots, x_{N^{\prime}}^{\prime}}, \psi_{x_{1}^{\prime}, \ldots, x_{N^{\prime}}^{\prime}}\right\rangle\right|-2 \frac{Z^{1 / 3}}{R^{2}} N^{\prime} \cdot\left\|\psi_{x_{1}^{\prime}, \ldots, x_{N^{\prime}}^{\prime}}\right\|_{2}^{2}\right)_{\left.d x_{1}^{\prime} \cdots d x_{N^{\prime}}^{\prime}\right)_{+}^{2}}\right. \\
& \leqq\left(\int\left(\left|\left\langle\sum_{j} T_{4} \psi_{x_{1}^{\prime}, \ldots, x_{N^{\prime}}^{\prime}}, \psi_{x_{1}^{\prime}, \ldots, x_{N^{\prime}}^{\prime}}\right\rangle\right|-2 \frac{Z^{1 / 3}}{R^{2}} N^{\prime} \cdot\left\|\psi_{x_{1}^{\prime}, \ldots, x_{N^{\prime}}}\right\|_{2}^{2}\right)_{+} d x_{1}^{\prime} \cdots d x_{N^{\prime}}^{\prime}\right)^{2} \\
& \leqq\left(\int\left\|\psi_{x_{1}^{\prime}, \ldots, x_{N^{\prime}}^{\prime}}\right\|_{2}^{2} d x_{1}^{\prime} \ldots d x_{N^{\prime}}^{\prime}\right)
\end{aligned}
$$




$$
\begin{aligned}
& \cdot \int_{E} \frac{\left(\left|\left\langle\sum_{j} T_{4} \psi_{i_{1}^{\prime}, \ldots, i_{N^{\prime}}^{\prime}}, \psi_{r_{1}^{\prime}, \ldots, i_{N^{\prime}}^{\prime}}\right\rangle\right|-2 \frac{Z^{1 / 3}}{R^{2}} N^{\prime} \cdot\left\|\psi_{r_{1}^{\prime}, \ldots, r_{N^{\prime}}^{\prime}}\right\|_{2}^{2}\right)_{+}^{2}}{\left\|\psi_{r_{1}^{\prime}, \ldots, x_{N^{\prime}}^{\prime}}\right\|_{2}^{2}} d x_{1}^{\prime} \ldots d x_{N^{\prime}}^{\prime} \\
& =\int_{E} \frac{\left(\left|\left\langle\sum_{j} T_{4} \psi_{r_{1}^{\prime}, \ldots, r_{N^{\prime}}^{\prime}}, \psi_{\lambda_{1}^{\prime}, \ldots, i_{N^{\prime}}^{\prime}}\right\rangle\right|-2 \frac{Z^{1 / 3}}{R^{2}} N^{\prime} \cdot\left\|\psi_{r_{1}^{\prime}, \ldots, i_{N^{\prime}}^{\prime}}\right\|_{2}^{2}\right)_{+}^{2}}{\left\|\psi_{r_{1}^{\prime} \ldots, r_{N^{\prime}}^{\prime}}\right\|_{2}^{2}} d x_{1}^{\prime} \ldots d x_{N^{\prime}}^{\prime} .
\end{aligned}
$$

Therefore

$$
\left\langle H_{Z, N} \psi, \psi\right\rangle \geqq E_{0}(Z)+C^{\prime} \frac{R^{2}}{N^{\prime 2} R_{*}}\left(\left|\left\langle\sum_{j} T_{4} \psi, \psi\right\rangle\right|-2 \frac{Z^{1 / 3}}{R^{2}} N^{\prime}\right)_{+}^{2}-C_{\mathrm{HSW}} Z^{7 / 3-b} .
$$

Recall that

$$
H_{Z, N}+H_{\text {extra }}=H_{Z, N+N^{\prime}}
$$

to obtain

$$
\begin{aligned}
\left\langle H_{Z, N+N^{\prime}} \psi, \psi\right\rangle \geqq & E_{0}(Z)+\left\langle H_{\text {extra }} \psi, \psi\right\rangle \\
& +C^{\prime} \frac{R^{2}}{N^{\prime 2} R_{*}}\left(\left|\left\langle\sum_{j} T_{4} \psi, \psi\right\rangle\right|-2 \frac{Z^{1 / 3}}{R^{2}} N^{\prime}\right)_{+}^{2}-C_{\mathrm{HSW}} Z^{7 / 3-b} .
\end{aligned}
$$

Using (3) we get

$$
\begin{aligned}
\left\langle H_{Z, N+N^{\prime}} \psi, \psi\right\rangle \geqq & E_{0}(Z)+\frac{\delta Z-20\left|Z-\left\langle N_{\chi} \psi, \psi\right\rangle\right|+\Omega}{9 R} N^{\prime}+\left\langle\sum_{j} T_{4} \psi, \psi\right\rangle \\
& +C^{\prime} \frac{R^{2}}{N^{\prime 2} R_{*}}\left(\left|\left\langle\sum_{j} T_{4} \psi, \psi\right\rangle\right|-2 \frac{Z^{1 / 3}}{R^{2}} N^{\prime}\right)_{+}^{2}-C_{\mathrm{HSW}} Z^{7 / 3-b} .
\end{aligned}
$$

Similarly, since

$$
\left\langle\mathrm{N}_{\chi} \psi, \psi\right\rangle=\int\left\langle N_{\chi} \psi_{x_{1}^{\prime}, \ldots, r_{N^{\prime}}^{\prime}}, \psi_{x_{1}^{\prime}, \ldots, x_{N^{\prime}}^{\prime}}\right\rangle d x_{1}^{\prime} \cdots d x_{N^{\prime}}^{\prime},
$$

if we let, for $\left(x_{1}^{\prime}, \ldots, x_{N^{\prime}}^{\prime}\right) \in E$,

$$
\left\langle N_{\chi} \bar{\psi}_{x_{1}^{\prime}, \ldots, x_{N^{\prime}}^{\prime}}, \bar{\psi}_{x_{1}^{\prime}, \ldots, x_{N^{\prime}}^{\prime}}\right\rangle=Z+Z^{1-\gamma_{1}} Y\left(x_{1}^{\prime}, \ldots, x_{N^{\prime}}^{\prime}\right)
$$

and

$$
\left\langle N_{\chi} \psi, \psi\right\rangle=Z+Y Z^{1-\gamma_{1}},
$$

then

$$
Y=\int_{E} Y\left(x_{1}^{\prime}, \ldots, x_{N^{\prime}}^{\prime}\right)\left\|\psi_{x_{1}^{\prime}, \ldots, x_{N^{\prime}}^{\prime}}\right\|_{2}^{2} d x_{1}^{\prime} \cdots d x_{N^{\prime}}^{\prime} .
$$

Now, Lemma 2.3 implies that

$$
\left\langle H_{Z, N} \bar{\psi}_{x_{1}^{\prime}, \ldots, x_{N^{\prime}}^{\prime}}, \bar{\psi}_{r_{1}^{\prime}, \ldots, x_{N^{\prime}}^{\prime}}\right\rangle \geqq E_{0}(Z)+\frac{C_{K}}{4} Z^{7 / 3-b}\left(\left|Y\left(x_{1}^{\prime}, \ldots, x_{N^{\prime}}^{\prime}\right)\right|-c_{1}\right)_{+}^{2}-C_{\mathrm{HSW}} Z^{7 / 3-b}
$$

for $\left(x_{1}^{\prime}, \ldots, x_{N^{\prime}}^{\prime}\right) \in E$. 
Arguing just as before we see that

$$
\begin{aligned}
\left(|Y|-c_{1}\right)_{+}^{2} & \leqq\left(\int_{E}\left(\left|Y\left(x_{1}^{\prime}, \ldots, x_{N^{\prime}}^{\prime}\right)\right|-c_{1}\right)\left\|\psi_{x_{1}^{\prime}, \ldots, x_{N^{\prime}}^{\prime}}\right\|_{2}^{2} d x_{1}^{\prime} \cdots d x_{N^{\prime}}^{\prime}\right)_{+}^{2} \\
& \leqq\left(\int_{E}\left(\left|Y\left(x_{1}^{\prime}, \ldots, x_{N^{\prime}}^{\prime}\right)\right|-c_{1}\right)_{+}\left\|\psi_{x_{1}^{\prime}, \ldots, x_{N^{\prime}}^{\prime}}\right\|_{2}^{2} d x_{1}^{\prime} \cdots d x_{N^{\prime}}^{\prime}\right)^{2} \\
& \leqq \int_{E}\left(\left|Y\left(x_{1}^{\prime}, \ldots, x_{N^{\prime}}^{\prime}\right)\right|-c_{1}\right)_{+}^{2}\left\|\psi_{x_{1}^{\prime}, \ldots, x_{N^{\prime}}^{\prime}}\right\|_{2}^{2} d x_{1}^{\prime} \cdots d x_{N^{\prime}}^{\prime}
\end{aligned}
$$

Therefore,

$$
\left\langle H_{Z, N} \psi, \psi\right\rangle \geqq E_{0}(Z)+\left(\frac{C_{K}}{4}\left(|Y|-c_{1}\right)_{+}^{2}-C_{\mathrm{HSW}}\right) Z^{7 / 3-b},
$$

and using (3) we see that

$$
\begin{gathered}
\left\langle H_{Z, N+N^{\prime}} \psi, \psi\right\rangle \geqq E_{0}(Z)+\frac{\delta Z-20\left|Z-\left\langle N_{\chi} \psi, \psi\right\rangle\right|+\Omega}{9 R} N^{\prime}+\left\langle\sum_{j} T_{4} \psi, \psi\right\rangle \\
+\left(\frac{C_{K}}{4}\left(|Y|-c_{1}\right)_{+}^{2}-C_{\text {HSw }}\right) Z^{7 / 3-b} .
\end{gathered}
$$

Therefore, averaging this expression with (5), we get that

$$
\begin{aligned}
\left\langle H_{Z, N+N^{\prime}} \psi, \psi\right\rangle \geqq & E_{0}(Z)+\frac{\delta Z-20\left|Z-\left\langle N_{\chi} \psi, \psi\right\rangle\right|+\Omega}{9 R} N^{\prime}+\left\langle\sum_{j} T_{4} \psi, \psi\right\rangle \\
& +\left(C^{\prime}\left(|Y|-c_{1}\right)_{+}^{2}-C_{\mathrm{HSW}}\right) Z^{7 / 3-b} \\
& +C^{\prime} \frac{R^{2}}{R_{*}}\left(\left|\frac{1}{N^{\prime}}\left\langle\sum_{j} T_{4} \psi, \psi\right\rangle\right|-2 \frac{Z^{1 / 3}}{R^{2}}\right)_{+}^{2}
\end{aligned}
$$

possibly with a different constant $C^{\prime}$.

Let's say now that, for some numbers $S$ and $V$,

$$
\begin{aligned}
\left\langle\sum_{j} T_{4} \cdot \psi, \psi\right\rangle & =-S \frac{\delta Z N^{\prime}}{R}, \\
N^{\prime} & =V \delta Z \frac{R}{R_{*}}>C_{0} V \delta Z .
\end{aligned}
$$

Thus $V \geqq 0$. Note that if $|S|<\frac{1}{16}$ and $|Y|$ is bounded above independently of $C_{0}$ we are done. This follows from the remarks following (3).

Observe that

$$
\begin{aligned}
\Omega & =N^{\prime}+N-Z-\delta Z \geqq\left(N^{\prime}+\left\langle N_{\chi} \psi, \psi\right\rangle-Z-\delta Z\right)_{+} \\
& \geqq\left(C_{0} V-\frac{2|Y|}{C_{0}}-1\right)_{+} \delta Z .
\end{aligned}
$$

Using (4) we can rewrite (6) to obtain

$$
\left\langle H_{Z, N+N^{\prime}} \psi, \psi\right\rangle \geqq E_{0}(Z)+F(S, Y, V) \cdot Z^{7 / 3-b}
$$


for

$$
\begin{aligned}
F(S, Y, V)= & \frac{C_{0}^{2} V}{9}-\frac{20}{9}\left(1+10^{-6}\right) C_{0} V|Y|+\left(\frac{C_{0}^{3} V^{2}-2 C_{0} V|Y|-C_{0}^{2} V}{9}\right)_{+} \\
& -\left(1+10^{-6}\right)^{2} C_{0}^{2}|S| V+C^{\prime}\left(\left(|Y|-c_{1}\right)_{+}^{2}+\left(C_{0}|S|-10^{-6}\right)_{+}^{2}\right)-C_{\mathrm{HSw}} \\
\geqq & \frac{1}{9}\left(C_{0}^{2} V-30 C_{0} V|Y|+\left(C_{0}^{3} V^{2}-2 C_{0} V|Y|-C_{0}^{2} V\right)_{+}-10 C_{0}^{2}|S| V\right. \\
& \left.+C^{\prime \prime}\left(\left(|Y|-c_{1}\right)_{+}^{2}+\left(C_{0}|S|-10^{-6}\right)_{+}^{2}\right)-9 C_{\mathrm{HSw}}\right) .
\end{aligned}
$$

Observe that we can assume that $C^{\prime \prime}<1$ and $c_{1}$ is so big that

$$
c_{1}^{2} C^{\prime \prime}-18 C_{\mathrm{HSW}}>2 \text {. }
$$

The rest of the lemma is devoted to proving that either $|S|<\frac{1}{16}$ and $|Y|<$ some constant or else $F>$ some other constant. In order to understand why this is so, we deal with different cases:

Case 1. $|S|>\frac{1}{16}|Y|<2 c_{1} \quad V<\frac{C^{\prime \prime}}{24}|S|$.

Since $|S|>\frac{1}{16}$, for $C_{0}>64$ we have $\left(C_{0}|S|-10^{-6}\right)_{+}^{2}>\frac{1}{2} C_{0}^{2} S^{2}$. Thus,

$$
\begin{aligned}
9 F & \geqq-\frac{5}{2} C^{\prime \prime} c_{1} C_{0}|S|-\frac{5}{12} C^{\prime \prime} C_{0}^{2} S^{2}+\frac{1}{2} C^{\prime \prime} C_{0}^{2} S^{2}-9 C_{\mathrm{HSW}} \\
& \geqq-40 C^{\prime \prime} c_{1} C_{0} S^{2}+\frac{1}{12} C^{\prime \prime} C_{0}^{2} S^{2}-9 C_{\mathrm{HSW}} \\
& \geqq C^{\prime \prime} C_{0} S^{2}\left(-40 c_{1}+\frac{1}{12} C_{0}\right)-9 C_{\mathrm{HSW}} \\
& \geqq \frac{C^{\prime \prime}}{16^{2}} C_{0}\left(-40 c_{1}+\frac{1}{12} C_{0}\right)-9 C_{\mathrm{HSW}},
\end{aligned}
$$

and pick $C_{0}$ large enough so that this is at least 1.

Case 2. $|S|>\frac{1}{16}|Y|<2 c_{1} V \geqq \frac{C^{\prime \prime}}{24}|S|$.

$$
9 F \geqq-60 c_{1} V C_{0}+\left(C_{0}^{3} V^{2}-4 c_{1} C_{0} V-C_{0}^{2} V\right)_{+}-10 V C_{0}^{2}|S|-9 C_{\mathrm{HSW}} .
$$

Pick now $C_{0}$ large enough so that

$$
\left(C_{0}^{3} V^{2}-4 c_{1} C_{0} V-C_{0}^{2} V\right)_{+}>\frac{1}{2} C_{0}^{3} V^{2} \text { for } V>\frac{C^{\prime \prime}}{24 \cdot 16}
$$

then

$$
\begin{aligned}
9 F & \geqq-60 c_{1} V C_{0}+\frac{1}{2} C_{0}^{3} V^{2}-\frac{240}{C^{\prime \prime}} V^{2} C_{0}^{2}-9 C_{\mathrm{HSW}} \\
& \geqq V^{2}\left(\frac{-60 \cdot 24 \cdot 16 c_{1}}{C^{\prime \prime}} C_{0}+\frac{1}{2} C_{0}^{3}-\frac{240}{C^{\prime \prime}} C_{0}^{2}\right)-9 C_{\mathrm{HSW}},
\end{aligned}
$$

and again pick $C_{0}$ large enough so that this is at least 1 for $V>C^{\prime \prime} / 24 \cdot 16$.

Case 3. All $|S||Y|>2 c_{1} V<10^{-6} C^{\prime \prime}$.

If $|S| \geqq \frac{1}{16}$, observe that $-10 C_{0}^{2} V|S|+C^{\prime \prime} / 2 C_{0}^{2}|S|^{2}$ is increasing in $|S|$ as long as 
$V<C^{\prime \prime} / 160$, which is true in our case. So it is enough to consider the case $|S|<\frac{1}{16}$. Observe that

$$
\begin{aligned}
& C_{0}^{2} V-10 C_{0}^{2} V|S| \geqq \frac{3}{8} C_{0}^{2} V \quad \text { if } \quad|S|<\frac{1}{16} \\
& \left(|Y|-c_{1}\right)_{+}^{2} \geqq \frac{Y^{2}}{4} \quad \text { if } \quad|Y| \geqq 2 c_{1} .
\end{aligned}
$$

Therefore

$$
\begin{aligned}
9 F & \geqq \frac{3}{8} C_{0}^{2} V-30 C_{0} V|Y|+\frac{C^{\prime \prime}}{4} Y^{2}-9 C_{\mathrm{HSw}} \\
& =\frac{3}{8} C_{0}^{2} V+\left(\sqrt{\frac{C^{\prime \prime}}{4}} Y-\frac{15 C_{0}}{\sqrt{C^{\prime \prime} / 4}} V\right)^{2}-\frac{900}{C^{\prime \prime}} C_{0}^{2} V^{2}-9 C_{\mathrm{HSw}} \\
& \geqq C_{0}^{2}\left(\frac{3}{8} V-\frac{900}{C^{\prime \prime}} V^{2}\right)-9 C_{\mathrm{HSw}} \\
& \geqq C_{0}^{2}\left(\frac{3}{8} V-\frac{900}{C^{\prime \prime}} 10^{-6} C^{\prime \prime} V\right)-9 C_{\mathrm{HSw}} \\
& \geqq C_{0}^{2} V\left(\frac{3}{8}-900 \cdot 10^{-6}\right)-9 C_{\mathrm{HSw}} .
\end{aligned}
$$

Now, if $V>100 C_{\mathrm{HSW}} C_{0}^{-2}$ we are done. Otherwise,

$$
\begin{aligned}
9 F & \geqq-3000 C_{\mathrm{HSW}} C_{0}^{-1}|Y|+\frac{C^{\prime \prime}}{4} Y^{2}-9 C_{\mathrm{HSW}} \\
& \geqq Y^{2}\left(-\frac{3000 C_{\mathrm{HSW}}}{2 c_{1} C_{0}}+\frac{C^{\prime \prime}}{4}\right)-9 C_{\mathrm{HSW}} \\
& \geqq \frac{C^{\prime \prime}}{8} Y^{2}-9 C_{\mathrm{HSW}} \geqq \frac{c_{1}^{2} C^{\prime \prime}}{2}-9 C_{\mathrm{HSW}} \geqq 1
\end{aligned}
$$

by (7) and for $C_{0}$ large enough.

Case 4. $|S|<\frac{1}{16}|Y|>2 c_{1} 10^{-13} C^{\prime 2} C_{0}^{1 / 3} \geqq V \geqq 10^{-6} C^{\prime \prime}$.

If $|Y|>C_{0}^{5 / 3}$, by (8)

$$
\begin{aligned}
9 F & \geqq-30 C_{0} V|Y|+\frac{C^{\prime \prime}}{4} Y^{2}-9 C_{\mathrm{HSW}} \\
& \geqq-3 \cdot 10^{-12} C^{\prime \prime 2} C_{0}^{4 / 3}|Y|+\frac{C^{\prime \prime}}{4} Y^{2}-9 C_{\mathrm{HSW}} .
\end{aligned}
$$

Differentiate with respect to $|Y|$ to realize that for $C_{0}>216 \cdot 10^{-36} C^{\prime \prime 3}$ the right-hand side is increasing for $|Y| \geqq C_{0}^{5 / 3}$. So,

$$
9 F \geqq-3 \cdot 10^{-12} C^{\prime 2} C_{0}^{9 / 3}+\frac{C^{\prime \prime}}{4} C_{0}^{10 / 3}-9 C_{\mathrm{HSw}},
$$

and pick $C_{0}$ big enough so that this is at least 1 . If, on the contrary, $|Y| \leqq C_{0}^{5 / 3}$, 
we have

$$
2 C_{0} V|Y|+C_{0}^{2} V \leqq 10^{-13} C^{\prime 2}\left(2 C_{0}^{3}+C_{0}^{7 / 3}\right) \leqq 3 \cdot 10^{-13} C^{\prime 2} C_{0}^{3} \leqq \frac{3}{10} C_{0}^{3} V^{2} .
$$

So, using (8) again

$$
9 F \geqq-30 C_{0} V|Y|+\frac{1}{2} C_{0}^{3} V^{2}-9 C_{\mathrm{HSw}} \geqq-30 C_{0}^{8 / 3} V+\frac{1}{2} C_{0}^{3} V^{2}-9 C_{\mathrm{HSW}},
$$

and pick $C_{0}$ so that this is bigger than 1 for $V \geqq 10^{-6} C^{\prime \prime}$.

Case 5. $|S| \geqq \frac{1}{16}|Y|>2 c_{1} 10^{-13} C^{\prime 2} C_{0}^{1 / 3} \geqq V \geqq 10^{-6} C^{\prime \prime}$.

In this case, argue as in Case 4 , with the only difference that $C_{0}^{2}\left(V-10|S| V+\frac{1}{2} C^{\prime \prime} S^{2}\right)$ need no longer be positive, and we have to include it in (9) and (10), which will be replaced respectively by

$$
9 F \geqq-3 \cdot 10^{-12} C^{\prime 2} C_{0}^{9 / 3}+\frac{C^{\prime \prime}}{4} C_{0}^{10 / 3}+C_{0}^{2}\left(V-10|S| V+\frac{1}{2} C^{\prime \prime} S^{2}\right)-9 C_{\mathrm{HSW}}
$$

and

$$
9 F \geqq-30 C_{0}^{8 / 3} V+\frac{1}{2} C_{0}^{3} V^{2}+C_{0}^{2}\left(V-10|S| V+\frac{1}{2} C^{\prime \prime} S^{2}\right)-9 C_{\mathrm{HSW}} .
$$

Note that

$$
C_{0}^{2}\left(V-10|S| V+\frac{1}{2} C^{\prime \prime} S^{2}\right) \geqq-\frac{100}{C^{\prime \prime}} C_{0}^{2} V^{2},
$$

since, for given $V$,

$$
\min _{|S|}\left(V-10|S| V+\frac{1}{2} C^{\prime \prime}|S|^{2}\right)
$$

is attained when $10 \mathrm{~V}=C^{\prime \prime}|S|$ : so,

$$
\min _{|S|}\left(V-10|S| V+\frac{1}{2} C^{\prime \prime}|S|^{2}\right) \geqq-\frac{100}{C^{\prime \prime}} V^{2} .
$$

Therefore, in our range of $V,(11)$ is at least $-10^{-24} C^{\prime \prime 3} C_{0}^{8 / 3}$, so, for $C_{0}$ big enough it doesn't affect the result since $V$ is bounded below by a constant independent of $C_{0}$.

Case 6. All $|S||Y|>2 c_{1} 10^{-13} C^{\prime 2} C_{0}^{1 / 3} \leqq V$.

If $|Y|>\left(200 / C^{\prime \prime}\right) C_{0} V$, and $|S|<\frac{1}{16}$, by (8),

$$
9 F \geqq-30 C_{0} V|Y|+\frac{C^{\prime \prime}}{4} Y^{2}-9 C_{\mathrm{HSW}}
$$

Again the derivative of this with respect to $|Y|$ is positive for $|Y|>\left(200 / C^{\prime \prime}\right) C_{0} V$. So, plugging in for $|Y|$ the value $|Y|=\left(200 / C^{\prime \prime}\right) C_{0}, V$, we obtain

$$
9 F \geqq C_{0}^{2} V^{2}\left(-\frac{6000}{C^{\prime \prime}}+\frac{10,000}{C^{\prime \prime}}\right)-9 C_{\mathrm{HSW}} \geqq \frac{4000}{C^{\prime \prime}} C_{0}^{2} V^{2}-9 C_{\mathrm{HSW}} .
$$

If $|S| \geqq \frac{1}{16}$, by (11) we have to subtract $\left(100 / C^{\prime \prime}\right) C_{0}^{2} V^{2}$, that does not alter the result. Now, if $|Y| \leqq\left(200 / C^{\prime \prime}\right) C_{0} V$, observe that

$$
C_{0}^{3} V^{2}-2 C_{0} V|Y|-C_{0}^{2} V \geqq C_{0}^{3} V^{2}-\frac{400}{C^{\prime \prime}} C_{0}^{2} V^{2}-C_{0}^{2} V \geqq \frac{1}{2} C_{0}^{3} V^{2}
$$


for $C_{0}$ big enough, since $V \geqq 1$. So, for $|S|<\frac{1}{16}$ we have

$$
\begin{aligned}
9 F & \geqq-30 C_{0} V|Y|+\frac{1}{2} C_{0}^{3} V^{2}-9 C_{\mathrm{HSW}} \\
& \geqq-\frac{6000}{C^{\prime \prime}} C_{0}^{2} V^{2}+\frac{1}{2} C_{0}^{3} V^{2}-9 C_{\mathrm{HSW}},
\end{aligned}
$$

and as usual we pick $C_{0}$ so that this is at least 1 . If $|S| \geqq \frac{1}{16}$, we have to subtract $\left(100 / C^{\prime \prime}\right) C_{0}^{2} V^{2}$, which again is harmless for $C_{0}$ big enough. This proves that either both $|S| \leqq \frac{1}{16}$ and $|Y| \leqq 2 c_{1}$ or

$$
\left\langle H_{Z, N+N^{\prime}} \psi, \psi\right\rangle \geqq E_{0}(Z)+c Z^{7 / 3-b}
$$

which concludes the lemma.

\section{The Bootstrap}

In Sect. two we obtained estimates for wave functions supported on balls, where the ball was to be of a certain size. The estimates from the previous section will help us obtain essentially the same kind of estimates for a ball of twice their size, and by induction, to all balls in $\mathbf{R}^{3}$.

Lemma 4.1. Let $R \geqq C_{0} Z^{-1 / 3+\gamma_{2}}, \bar{\varepsilon}=c_{0} Z^{-\gamma_{1}}$ and

$$
C_{0} Z^{-\gamma_{1}} \leqq \varepsilon \leqq\left(1+10^{-12}\right) C_{0} Z^{-\gamma_{1}} \text {. }
$$

If Estimate $(\bar{\varepsilon}, \varepsilon, R)$ holds, then Estimate $\left(\bar{\varepsilon}, \varepsilon^{\prime}, 2 R\right)$ also holds, with

$$
\varepsilon^{\prime}=\varepsilon+\frac{2 C}{\bar{\varepsilon} R Z}
$$

provided that $\varepsilon^{\prime} \leqq \varepsilon^{\#}<1$.

Proof. We consider a partition of unity given by two smooth functions, $\theta_{0}$ and $\theta_{1}$, satisfying

$$
\begin{gathered}
\theta_{0}(x)=\left\{\begin{array}{lll}
1 & \text { if } & |x|<R / 2 \\
0 & \text { if } & |x|>R
\end{array},\right. \\
\theta_{0}^{2}(x)+\theta_{1}^{2}(x)=1 .
\end{gathered}
$$

Given a wave function $\psi\left(x_{1}, \ldots, x_{N}\right)$ supported on $B(0,2 R)$, and given any sequence $i_{1}, \ldots, i_{N}$ of 0 's and 1's, we define

$$
\psi_{i_{1}, \ldots, i_{N}}=\theta_{i_{1}}\left(x_{1}\right) \cdots \theta_{i_{N}}\left(x_{N}\right) \psi\left(x_{1}, \ldots, x_{N}\right) .
$$

Assume for simplicity that $i_{j}=0$ for $j=1, \ldots, N_{1}$ and $i_{j}=1$ for $j=N_{1}+1, \ldots, N$; let $N_{2}=N-N_{1}$. We define $\psi_{x_{N_{1}+1}, \ldots, x_{N}}$ to be $\psi$, where the variables $x_{N_{1}+1}, \ldots, x_{N}$ are fixed. It is thus an antisymmetic wave function supported on $(B(0, R))^{N_{1}}$.. Since Estimate $(\bar{\varepsilon}, \varepsilon, R)$ holds, we have

$$
\left\langle H_{Z, N_{1}} \psi_{x_{N_{1}+1}, \ldots, x_{N}}, \psi_{x_{N_{1}+1}, \ldots, x_{N}}\right\rangle \geqq\left(E_{0}(Z)+\frac{\bar{\varepsilon} Z}{R}\left(N_{1}-(1+\varepsilon) Z\right)\right)\left\|\psi_{x_{N_{1}+1}, \ldots, x_{N}}\right\|_{2}^{2} .
$$


Integrate this against $d x_{N_{1}+1}, \ldots, d x_{N}$ to obtain

$$
\left\langle H_{Z, N_{1}} \psi_{i_{1}, \ldots, i_{N}}, \psi_{i_{1}, \ldots, i_{N}}\right\rangle \geqq\left(E_{0}(Z)+\frac{\bar{\varepsilon} Z}{R}\left(N_{1}-(1+\varepsilon) Z\right)\right)\left\|\psi_{i_{1}, \ldots, i_{N}}\right\|_{2}^{2} .
$$

Our goal now is to prove that

$$
\begin{aligned}
& \left\langle H_{Z, N_{1}+N_{2}} \psi_{i_{1}, \ldots, i_{N}}, \psi_{i_{1}, \ldots, i_{N}}\right\rangle \\
& \quad \geqq\left(E_{0}(Z)+\frac{\bar{\varepsilon} Z}{R}\left(N_{1}+N_{2}-(1+\varepsilon) Z\right)\right)\left\|\psi_{i_{1}, \ldots, i_{N}}\right\|_{2}^{2} .
\end{aligned}
$$

This is trivial if $N=N_{1}+N_{2} \leqq(1+\varepsilon) Z$, since $\varepsilon \leqq \varepsilon^{\#}$. If $N \geqq(1+\varepsilon) Z$, we can apply Lemma 3.1, with $\delta=\varepsilon$. If

$$
\left\langle H_{Z, N_{1}+N_{2}} \psi_{i_{1}, \ldots, i_{N}}, \psi_{i_{1}, \ldots, i_{N}}\right\rangle \geqq\left(E_{0}(Z)+c Z^{7 / 3-b}\right)\left\|\psi_{i_{1}, \ldots, i_{N}}\right\|_{2}^{2},
$$

then, either

$$
c Z^{7 / 3-b}>\frac{\bar{\varepsilon} Z}{R}(N-(1+\varepsilon) Z)
$$

in which case (13) is proved, or

$$
c Z^{7 / 3-b} \leqq \frac{\bar{\varepsilon} Z}{R}(N-(1+\varepsilon) Z)
$$

If this is the case, just like in (2), note that provided $c_{0}<c C_{\mathrm{K}} /\left(8 C_{\mathrm{HSW}}\right)$, where $c$ is the constant in Lemma 3.1 (we can assume $c<1$ and $C_{\mathrm{HSW}}>1$ ),

$$
c C_{\mathrm{K}} \frac{|N-Z|^{2}}{8 C_{\mathrm{HSW}} R}>\frac{\bar{\varepsilon} Z}{R}(N-(1+\varepsilon) Z) .
$$

Equation (14) then implies that

$$
C_{\mathrm{K}} \frac{|N-Z|^{2}}{4 R}>2 C_{\mathrm{HSW}} Z^{7 / 3-b} .
$$

Estimate (A) then with $\chi=\chi_{2 R}$ implies that

$$
\begin{aligned}
\left\langle H_{Z, N_{1}+N_{2}} \psi_{i_{1}, \ldots, i_{N}}, \psi_{i_{1}, \ldots, i_{N}}\right\rangle & \geqq\left(E_{0}(Z)+C_{\mathrm{K}} \frac{|N-Z|^{2}}{8 R}\right)\left\|\psi_{i_{1}, \ldots, i_{N}}\right\|_{2}^{2} \\
& \geqq\left(E_{0}(Z)+c C_{\mathrm{K}} \frac{|N-Z|^{2}}{8 C_{\mathrm{HSW}} R}\right)\left\|\psi_{i_{1}, \ldots, i_{N}}\right\|_{2}^{2},
\end{aligned}
$$

and (15) again implies (13).

The alternative left from Lemma 3.1 is that

$$
\left\langle H_{\mathrm{extra}} \psi_{i_{1}, \ldots, i_{N}}, \psi_{i_{1}, \ldots, i_{N}}\right\rangle \geqq \frac{c \varepsilon Z}{R} N_{2}\left\|\psi_{i_{1}, \ldots, i_{N}}\right\|_{2}^{2} .
$$

Since

$$
H_{Z, N_{1}+N_{2}}=H_{Z, N_{1}}+H_{\text {extra }}
$$


and $c \varepsilon>\bar{\varepsilon}$ (for $c_{0}<c \cdot C_{0}$ ), (12) and (16) imply

$$
\left\langle H_{Z, N_{1}+N_{2}} \psi_{i_{1}, \ldots, i_{N}}, \psi_{i_{1}, \ldots, i_{N}}\right\rangle \geqq\left(E_{0}(Z)+\frac{\bar{\varepsilon} Z}{R}\left(N_{1}+N_{2}-(1+\varepsilon) Z\right)\right) \cdot\left\|\psi_{i_{1}, \ldots, i_{N}}\right\|_{2}^{2},
$$

and (13) is proved.

Putting all these estimates together, we see that

$$
\sum_{i_{1}, \ldots, i_{N}}\left\langle H_{Z, N_{1}+N_{2}} \psi_{i_{1}, \ldots, i_{N}}, \psi_{i_{1}, \ldots, i_{N}}\right\rangle \geqq E_{0}(Z)+\frac{\bar{\varepsilon} Z}{R}\left(N_{1}+N_{2}-(1+\varepsilon) Z\right) .
$$

Now,

$$
\sum_{i_{1}, \ldots, i_{N}}\left\langle V_{\text {Coulomb }} \psi_{i_{1}, \ldots, i_{N}}, \psi_{i_{1}, \ldots, i_{N}}\right\rangle=\left\langle V_{\text {Coulomb }} \psi, \psi\right\rangle .
$$

For $-\Delta$, we have

$$
\begin{aligned}
& \sum_{i_{1}, \ldots, i_{N}}\left\langle-\Delta_{x_{1}, \ldots, x_{N}} \psi_{i_{1}, \ldots, i_{N}}, \psi_{i_{1}, \ldots, i_{N}}\right\rangle \\
& =\sum_{i_{1}, \ldots, i_{N}} \sum_{k}\left\langle-\Delta_{x_{k}} \psi_{i_{1}, \ldots, i_{N}}, \psi_{i_{1}, \ldots, i_{N}}\right\rangle \\
& =\sum_{i_{1}, \ldots, i_{N}} \sum_{k}\left\langle\prod_{j \neq k} \theta_{i j}^{2}\left(x_{j}\right) \cdot\left(-\Delta_{x_{k}} \theta_{i_{k}}\left(x_{k}\right) \psi\right),\left(\theta_{x_{k}}\left(x_{k}\right) \psi\right)\right\rangle \\
& =\sum_{k} \sum_{i_{k}}\left\langle-\Delta_{x_{k}}\left(\theta_{i_{k}}\left(x_{k}\right) \psi\right),\left(\theta_{i_{k}}\left(x_{k}\right) \psi\right)\right\rangle \\
& =\sum_{i, k}\left\langle\theta_{i}^{2}\left(x_{k}\right)\left(-\Delta_{x_{k}} \psi\right), \psi\right\rangle+\sum_{i, k}\left\langle\psi\left(-\Delta_{x_{k}} \theta_{i}\left(x_{k}\right)\right), \theta_{i}\left(x_{k}\right) \psi\right\rangle \\
& \quad-2 \sum_{i, k}\left\langle\nabla_{x_{k}} \psi \cdot \nabla_{x_{k}} \theta_{i}\left(x_{k}\right), \psi \theta_{i}\left(x_{k}\right)\right\rangle \\
& =\langle-\Delta \psi, \psi\rangle+\left\langle\sum_{i, k} \theta_{i}\left(x_{k}\right)\left(-\Delta_{x_{k}} \theta_{i}\left(x_{k}\right)\right) \psi, \psi\right\rangle-\sum_{i, k}\left\langle\nabla_{x_{k}} \theta_{i}^{2}\left(x_{k}\right) \cdot \nabla_{x_{k}} \psi, \psi\right\rangle .
\end{aligned}
$$

The last term on the right is zero, since

$$
\sum_{i} \nabla_{x_{k}} \theta_{i}^{2}\left(x_{k}\right)=\nabla_{x_{k}} \sum_{i} \theta_{i}^{2}\left(x_{k}\right)=\nabla 1=0 .
$$

Hence, if we define

$$
W(x)=\sum_{i} \theta_{i}(x) \Delta \theta_{i}(x)
$$

we get

$$
\sum_{i_{1}, \ldots, i_{N}}\left\langle-\Delta_{x_{1}, \ldots, x_{N}} \psi_{i_{1}, \ldots, i_{N}}, \psi_{i_{1}, \ldots, i_{N}}\right\rangle=\langle-\Delta \psi, \psi\rangle-\left\langle\sum_{k} W\left(x_{k}\right) \psi, \psi\right\rangle .
$$

As result, we get

$$
\left\langle H_{Z, N} \psi, \psi\right\rangle-\left\langle\sum_{k} W\left(x_{k}\right) \psi, \psi\right\rangle \geqq E_{0}(Z)+\frac{\bar{\varepsilon} Z}{R}(N-(1+\varepsilon) Z) .
$$

Observe now that

$$
|W(x)| \leqq \frac{C}{R^{2}} .
$$


Thus it follows that

$$
\left|\left\langle\sum_{k} W\left(x_{k}\right) \psi, \psi\right\rangle\right| \leqq \frac{C N}{R^{2}}
$$

Thus we have

$$
\left\langle H_{Z, N} \psi, \psi\right\rangle \geqq E_{0}(Z)+\frac{\bar{\varepsilon} Z}{R}(N-(1+\varepsilon) Z)-\frac{C N}{R^{2}} .
$$

So we have only left to investigate when

$$
\frac{\bar{\varepsilon} Z}{R}(N-(1+\varepsilon) Z)-\frac{C N}{R^{2}} \geqq \frac{\bar{\varepsilon} Z}{2 R}\left(N-\left(1+\varepsilon^{\prime}\right) Z\right) .
$$

Observe that the derivative with respect to $N$ of the left-hand side is bigger than the derivative of the right-hand side. This amounts to checking that

$$
\frac{\bar{\varepsilon} Z}{R}-\frac{C}{R^{2}} \geqq \frac{\bar{\varepsilon} Z}{2 R},
$$

which is equivalent to

$$
\frac{C}{R} \leqq \frac{\bar{\varepsilon} Z}{2}
$$

which will hold as long as $R \geqq 2 C / \bar{\varepsilon} Z$. This certainly holds in our case, since

$$
R>Z^{-1 / 3+\gamma_{2}} \gg Z^{-1+\gamma_{1}} \text {. }
$$

So it is enough to prove that (17) holds for the smallest value of $N$ in which we are interested. For $N=\left(1+\varepsilon^{\prime}\right) Z,(17)$ is equivalent to

$$
C \frac{1+\varepsilon^{\prime}}{R} \leqq \bar{\varepsilon} Z\left(\varepsilon^{\prime}-\varepsilon\right)
$$

i.e.

$$
\varepsilon^{\prime} \geqq \varepsilon+\frac{C\left(1+\varepsilon^{\prime}\right)}{\bar{\varepsilon} Z R}
$$

So, Estimate $\left(\bar{\varepsilon}, \varepsilon^{\prime}, 2 R\right)$ holds with

$$
\varepsilon^{\prime}=\varepsilon+\frac{2 C}{\bar{\varepsilon} Z R}
$$

Corollary. There exist $\bar{\varepsilon}^{\#}, \varepsilon^{\#}$ such that Estimate $\left(\bar{\varepsilon}^{\#}, \varepsilon^{\#}, R\right)$ holds for all $R \geqq C_{0} Z^{-1 / 3+\gamma_{2}}$. Proof. Define

$$
\begin{aligned}
\varepsilon_{0} & =C_{0} Z^{-\gamma_{1}}, \quad \bar{\varepsilon}_{0}=c_{0} Z^{-\gamma_{1}} \\
R_{0} & =C_{0} Z^{-1 / 3+\gamma_{2}}, \quad \varepsilon_{n}=\varepsilon_{n-1}+\frac{2 C}{\bar{\varepsilon}_{0} Z R_{n}}, \quad R_{n}=2^{n} R_{0}
\end{aligned}
$$


Note that

$$
\varepsilon_{n} \leqq \varepsilon_{0}+\sum_{k=0}^{\infty} \frac{4 C}{\overline{\varepsilon_{0}} Z 2^{k} R_{0}}<C^{\#} \varepsilon_{0} \stackrel{\text { def }}{=} \varepsilon^{\#}
$$

for $Z$ large enough, provided $b<\frac{14}{15}$ (which is true in our discussion), with $C^{\#} \leqq\left(1+10^{-12}\right)$.

By Lemma 2.2 we see that Estimate $\left(\bar{\varepsilon}_{0}, \varepsilon_{0}, R_{0}\right)$ holds. Therefore, by the previous lemma, if we make $\bar{\varepsilon}^{\#}=\bar{\varepsilon}_{0}$, Estimate $\left(\bar{\varepsilon}_{0}, \varepsilon_{n}, R_{n}\right)$ holds for all $n$, and the corollary follows. This implies that

$$
\left\langle H_{Z, N} \psi, \psi\right\rangle \geqq E_{0}(Z)
$$

and therefore

$$
N(Z) \leqq Z+\varepsilon^{\#} Z=Z+O\left(Z^{1-\gamma_{1}}\right) .
$$

From [11] it follows that

$$
N(Z) \geqq Z,
$$

and therefore,

$$
N(Z)=Z+O\left(Z^{1-\gamma_{1}}\right)
$$

which proves the theorem.

\section{References}

1. Fefferman, C., Seco, L.: An upper bound for the number of electrons in a large ion, to appear in Proceedings of the Nat. Acad. Sci. USA (1989)

2. Hertel, P., Lieb, E., Thirring, W.: Lower bound to the energy of complex atoms. J. Chem. Phys. 62, 3355 (1975)

3. Hughes, W.: To appear in Adv. Math.

4. Lieb, E. H.: A lower bound for Coulomb energies. Phys. Lett. 70A, 444 (1979)

5. Lieb, E. H.: Thomas-Fermi and related theories of atoms and molecules. Rev. Mod. Phys. $\mathbf{5 3}$ (1981)

6. Lieb, E. H.: Atomic and molecular negative ions. Phys. Rev. Lett. 52, 315 (1984)

7. Lieb, E. H.: Bound on the maximum negative ionization of atoms and molecules. Phys. Rev. A29, 3018-3028 (1984)

8. Lieb, E. H., Sigal, I., Simon, B., Thirring, W.: Approximate neutrality of large- $Z$ ions. Commun. Math. Phys. 116, 635-644 (1988)

9. Siedentop, H., Weikard, R.: On the leading energy correction for the statistical model of the atom: interacting case. Commun. Math. Phys. 112, 471-490 (1987)

10. Siedentop, H., Weikard, R.: To appear in Invent. Math.

11. Zhislin, G.: Discussion of the spectrum of Schrödinger operator for system of many particles. Tr. Mosk. Mat. Obs. 9, 81-128 (1960)

Communicated by B. Simon

Received May 23, 1989; in revised form July 26, 1989 